\title{
Competition for FcRn-mediated transport gives rise to short half-life of human IgG3 and offers therapeutic potential
}

\author{
Nigel M. Stapleton ${ }^{1}$, Jan Terje Andersen ${ }^{2,3, \star}$, Annette M. Stemerding ${ }^{4, \star}$, Stefania P. Bjarnarson ${ }^{5}$, \\ Ruurd C. Verheul ${ }^{4}$, Jacoline Gerritsen ${ }^{1}$, Yixian Zhao ${ }^{6}$, Marion Kleijer $^{1}$, Inger Sandlie ${ }^{3}$, Masja de Haas ${ }^{1}$, \\ Ingileif Jonsdottir ${ }^{5}$, C. Ellen van der Schoot $^{1} \&$ Gestur Vidarsson ${ }^{1}$
}

Human IgG3 displays the strongest effector functions of all IgG subclasses but has a short half-life for unresolved reasons. Here we show that lgG3 binds to IgG-salvage receptor (FcRn), but that FcRn-mediated transport and rescue of $\operatorname{lgG} 3$ is inhibited in the presence of $\lg \mathrm{g} 1$ due to intracellular competition between IgG1 and IgG3. We reveal that this occurs because of a single amino acid difference at position 435, where lgG3 has an arginine instead of the histidine found in all other IgG subclasses. While the presence of R435 in IgG increases binding to FcRn at neutral $\mathrm{pH}$, it decreases binding at acidic $\mathrm{pH}$, affecting the rescue efficiency-but only in the presence of H435-IgG. Importantly, we show that in humans the half-life of the H435containing lgG3 allotype is comparable to lgG1. H435-lgG3 also gave enhanced protection against a pneumococcal challenge in mice, demonstrating $\mathrm{H} 435-\operatorname{lgG} 3$ to be a candidate for monoclonal antibody therapies.

\footnotetext{
${ }_{1}^{1}$ Department of Experimental Immunohematology, Sanquin Research, and Landsteiner Laboratory, Academic Medical Center, University of Amsterdam, Plesmanlaan 125, Amsterdam 1066 CX, The Netherlands. ${ }^{2}$ Department of Immunology, Oslo University Hospital Rikshospitalet and University of Oslo, PO Box 4950 Nydalen, Oslo 0424, Norway. ${ }^{3}$ Centre for Immune Regulation and Department of Molecular Biosciences, University of Oslo, PO box 1041 , Blindern, Oslo 0316, Norway. ${ }^{4}$ Department of Medical Microbiology, University Medical Center Utrecht, Heidelberglaan 100, 3584 CX, The Netherlands. 5 Department of Immunology, Landspitali University Hospital, Faculty of Medicine, University of Iceland, Eiriksgata, hus 14, Reykjavik 101, Iceland.

${ }^{6}$ Department of Pharmacology, Utrecht University, Universiteitsweg 99, Utrecht 3584 CG, The Netherlands. ${ }^{*}$ These authors contributed equally to this work. Correspondence and requests for materials should be addressed to G.V. (email: G.Vidarsson@sanquin.nl).
} 
H uman IgG3 activates complement and Fc $\gamma$ R-mediated functions more effectively than any other subclass, followed by IgG1, IgG2 and IgG4, respectively, making it an ideal candidate for immunotherapy ${ }^{1-4}$. However the short half-life of one week for IgG3, compared with three weeks for the other subclasses, currently makes IgG1 the therapeutic subclass of choice $e^{4,5}$.

The exceptionally long half-life of $\operatorname{IgG}$ is mediated by a single receptor, the neonatal Fc receptor for $\operatorname{IgG}(\mathrm{FcRn})^{6,7}$. FcRn is a heterodimer consisting of a unique MHC class-I like $\alpha$-chain, associated with $\beta 2 \mathrm{M}$. Because its affinity for IgG is negligible at physiological $\mathrm{pH}$ $(\sim 7.4)$, FcRn binds to IgG only after pinocytosis within early endosomes $(\mathrm{pH} \leq 6.5)^{8,9}$. FcRn-IgG complexes are then routed away from the lysosomal pathway ${ }^{10-13}$, and either cycled back to the cell surface or transported to the opposite side of the cell. The vesicles fuse with the plasma membrane, returning the $\mathrm{pH}$ to $\sim 7.4$, and releasing $\operatorname{IgG}^{14-16}$. Besides IgG transport, FcRn enhances IgG-mediated phagocytosis as well as antigen presentation by both MHC class I and II ${ }^{16-19}$, and has a key role in rescuing albumin from lysosomal degradation ${ }^{20}$.

Two theoretical explanations for the reduced half-life of IgG3 have been suggested. First, the long hinge region of IgG3 might make it more prone to proteolytic degradation ${ }^{4}$. Second, the recycling of IgG3 by FcRn may be less efficient because of an amino acid difference at position 435-a key contact residue with FcRn and, in IgG1, IgG2 and IgG4, important for the pH-dependent formation of IgG-FcRn complexes through histidine protonation around $\mathrm{pH} \leq 6.5$ (refs $8,9,21,22$ ). IgG3 contains an arginine in this position (R435), and although both residues classify as positively charged, arginines, unlike histidines, do not deprotonate at neutral $\mathrm{pH}$, theoretically resulting in IgG3 binding to FcRn being less $\mathrm{pH}$ dependent, leading to shortened half-life as described for various mutated IgG variants with increased affinity to FcRn at neutral $\mathrm{pH}$ (refs 8, 23). Previous experimental work indeed pointed to the involvement of R435, as human IgG1-Fc and scFv-IgG1-Fc fragments bearing R435 showed reduced half-life in wild-type mice ${ }^{24,25}$. Human H435R-IgG4 variant was also reported to display an altered binding to rat $\mathrm{FcRn}^{8}$. However, species-incompatible FcRn were used in these studies; the exceptional long hinge of IgG3 was not present; and the underlying mechanisms were not explored ${ }^{4,8,24-27}$.

Here we investigate the difference in FcRn-mediated transport and rescue of $\operatorname{IgG} 1$ and $\operatorname{IgG} 3$. Using human in vitro and in vivo models, we observed-unexpectedly-that both IgG1 and IgG3 show $\mathrm{pH}$-dependent binding to FcRn, and that FcRn can transport IgG3 as efficiently as IgG1. However, when both IgG1 and IgG3 are present, IgG1 inhibits FcRn-mediated IgG3 transport, leading to degradation of IgG3. Our data provide strong evidence that the presence of an arginine at position 435 in IgG3 is sufficient to explain its high rate of catabolism observed in vivo. Importantly, we show that the half-life of the H435-containing IgG3 allotype is comparable to IgG1 in humans. Using a mouse model for pneumococcal pneumonia, we provide a proof of concept that IgG3-R435H can be utilized for IgG-based immunotherapies aiming at maximizing effector functions.

\section{Results}

IgG1 interferes with IgG3 transport. To study FcRn-mediated functions, we developed an in vitro transport model by transducing the FcRn-negative human cell line A375 with the human FcRn $\alpha$-chain (A375-FcRn). The wild-type A375 did not transport IgG using intravenous immunoglobulin (IVIg), a polyclonal mixture of all human IgG subclasses (Fig. 1a). However, active transport was observed in A375-FcRn cells in medium buffered at $\mathrm{pH}$ 7.4. We found IgG transport in A375-FcRn to be similar to that observed across placental syncytiotrophoblast derived JAR cells expressing endogenous FcRn (Fig. 1b). From IVIg, A375-FcRn cells transported IgG3 less effectively than IgG1, but JAR transported relatively equal amounts of both IgG1 and IgG3 (Fig. 1a,b).
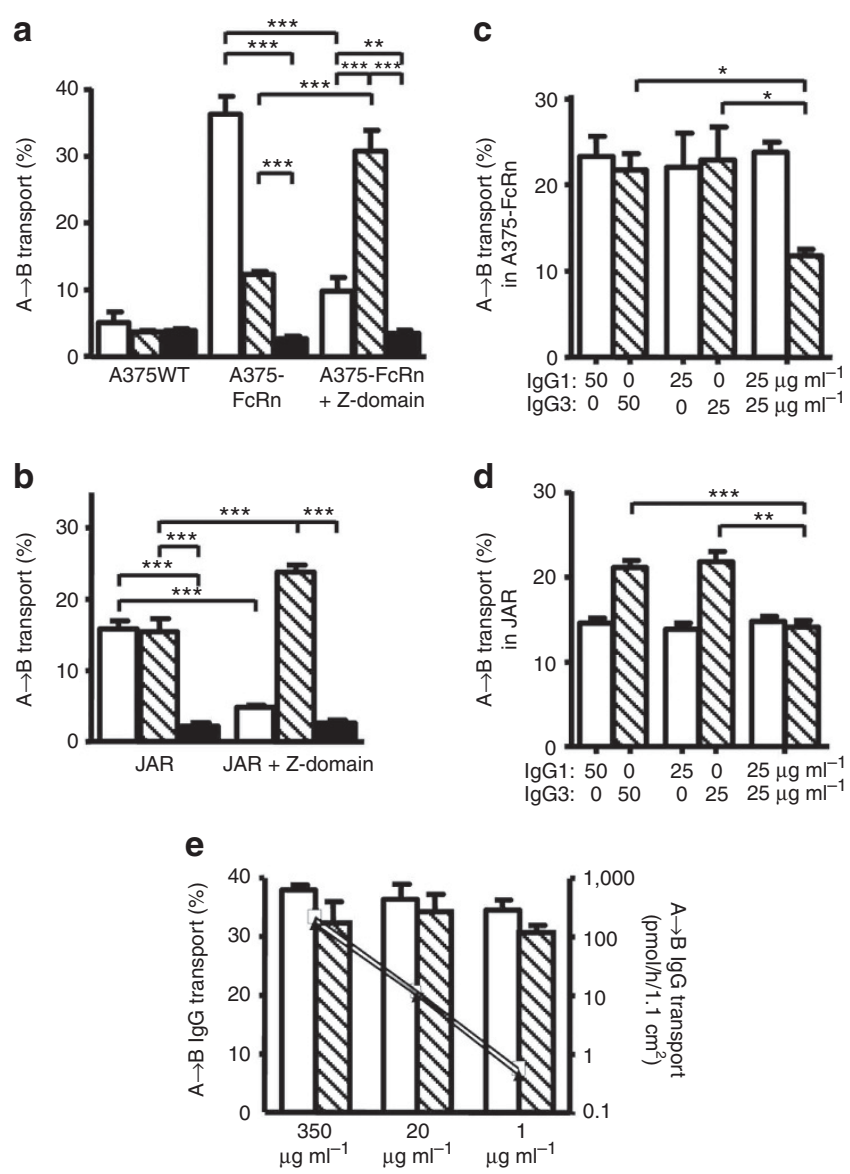

Figure 1 | IgG3 transport is inhibited by IgG1 at non-saturating conditions. All experiments were performed at $\mathrm{pH}$ 7.4. (a) FcRn-negative human A375-WT cells did not transcytose lgG1 (white) and lgG3 (hatched) from IVIg as transport was comparable to passive leakage (HRP, black). After transfection with the FcRn $\alpha$-chain, A375-FcRn efficiently transported IgG from the apical to the basolateral compartment. When IVIg was mixed with Z-domain before transport at a 2:1 molar ratio (Z-domain:lgG) the IgG1 transport by A375-FcRn cells was significantly reduced, while IgG3 transport was enhanced. (b) JAR cells naturally expressing FcRn transported IgG1 and IgG3 from IVIg equally well. Incubation of IVIg with the Z-domain at a 2:1 molar ratio before transport inhibited transport of IgG1 but increased transport of IgG3. (c) Purified IgG3 and IgG1 were transcytosed equally well in A375-FcRn cells when transported separately, and neither inhibited its own transport when the input was doubled. Yet in 1:1 mixtures, IgG3 transport was reduced in the presence of IgG1. (d) In JAR cells, IgG3 was efficiently transported when offered alone. The amount of either IgG1 or IgG3 transported was also unaffected by doubling the apical concentration, but lgG3 transport was inhibited by the presence of equal amounts of IgG1. (e) When only one subclass was present, A375-FcRn transported a fixed percentage of IgG (left axis), while the absolute amount transported was diminished (IgG1 open squares, IgG3 triangles, right axis). Throughout, IgG1 is represented by open bars, IgG3 by hatched bars. $100 \mu \mathrm{g} \mathrm{ml}^{-1} \mathrm{IVIg}$ was used in both (a,b). Apical to basolateral transport of myeloma lgG1 and IMlg-derived lgG3 in the concentration indicated in (c-e). The $Y$-axis represents the percentage of IgG transported from the apical compartment to the basolateral compartment. The data represent mean and standard deviation from three independent experiments. Statistical comparison was performed by one-way ANOVA followed by Tukey's multiple comparison test in (a,b), and transport of IgG3, in the presence of IgG1, was compared with transport of $\operatorname{lgG} 3$ alone by two-tailed $t$-test in $(\mathbf{c}, \mathbf{d}) .{ }^{\star} P \leq 0.05 ;{ }^{\star \star} P \leq 0.01$; ${ }^{\star \star \star} P \leq 0.001$. 


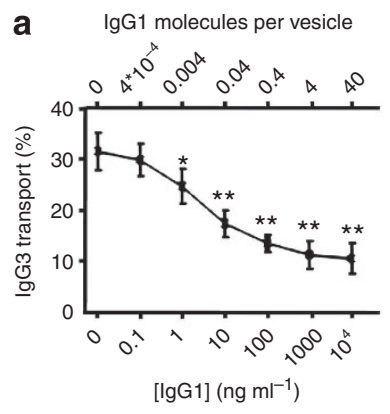

C

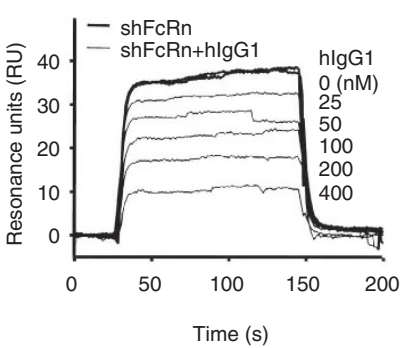

b IgG molecules per vesicle per subclass

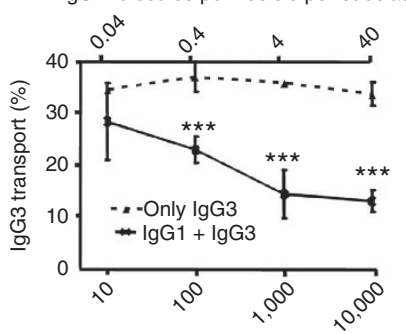

[lgG] (ng per ml per subclass)

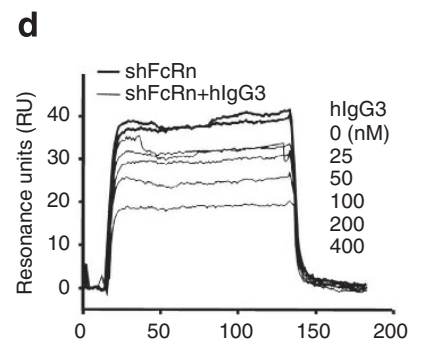

Time (s)

Figure $\mathbf{2}$ | Concentration-dependent inhibition of IgG3 transport by IgG1 owing to competitive binding to FcRn. Apical to basolateral transport of $\mathrm{V}$-gene matched recombinant IgG3 and IgG1. (a) IgG3 concentration was kept constant $\left(10 \mu \mathrm{g} \mathrm{ml}^{-1}\right)$ in the absence or presence of increasing amounts of IgG1. IgG3 transport was inhibited up to a plateau when more than $1 \mathrm{ng} \mathrm{ml} l^{-1} \mathrm{lgG} 1$ was present. All data points were compared with the samples without IgG1 by one-way ANOVA and Dunnett's multiple comparison test. (b) Recombinant lgG3 alone (dotted line) or mixed with IgG1 (solid line) at a 1:1 ratio in increasing concentrations were added to the apical compartment and lgG3 was measured in the basolateral compartment. At concentrations lower than $1 \mu \mathrm{g} \mathrm{ml}^{-1}$, transport of lgG3 increased up to levels similar to those observed when $\operatorname{lgG} 3$ is transported alone. All data points from mixed lgG1 and lgG3 transport in (b) were compared by $t$-test to the corresponding lgG3 transport without IgG1 present. The theoretical number of IgG molecules present in 1.25$\mu \mathrm{m}$-wide sorting endosomes described in ref. 15, assuming an equal concentration within these vesicles as present in the medium, is indicated on the secondary upper $x$-axis in $(\mathbf{a}, \mathbf{b})$. This arbitrary value is given as an indication only as these calculated values cannot take miscellaneously elongated or tubule-tethered vesicles into account ${ }^{13,15}(\mathbf{c}, \mathbf{d})$. Surface plasma resonance analysis showing binding of 100-nM recombinant shFcRn to IgG3-immobilized CM5 biosensor chips (1,300 RU) at pH 6.0 in the presence of increasing concentrations of $25,50,100,200$ or 400-nM recombinant soluble $\lg G 1$ (c) or $\operatorname{lgG} 3(\mathbf{d})$. The data in $(\mathbf{a}, \mathbf{b})$ represent the mean and standard deviation. All experiments were repeated at least three times with similar results. ${ }^{\star} P \leq 0.05 ;{ }^{\star \star} P \leq 0.01 ;{ }^{\star \star \star} P \leq 0.001$.

When IVIg was pre-incubated with Z-domains of protein A, a selective competitive inhibitor of FcRn binding to IgG1, IgG2 and IgG4 (refs 28, 29), transport of IgG1 by JAR and A375-FcRn cells was reduced to levels approaching those found in parental A375-WT and for non-specific horseradish peroxidase (HRP) transport and (Fig. 1), indicating IgG1 transport to be FcRn-dependent in both cell types. Remarkably, IgG3 transport was significantly increased in both JAR and A375-FcRn cells by the addition of Z-domains (Fig. 1a,b), suggesting that active transport of the other IgG subclasses interfered with IgG3 transport.

We therefore studied the effect of IgG1 on the FcRn-mediated transport of IgG3 using purified antibodies. Indeed, IgG3 alone was transported equally well as IgG1, and the transport of IgG3 was inhibited by adding IgG1 to A375-FcRn (Fig. 1c) or JAR

cells (Fig. 1d). As the percentage of IgG1 or IgG3 transport was unaffected by the initial IgG concentration (measured over a range from 1 to $350 \mu \mathrm{g} \mathrm{ml}^{-1}$; Fig. 1e) the observed inhibition of IgG3 transport by $\operatorname{IgG1}$ (at concentrations of $50-100 \mu \mathrm{g} \mathrm{ml}^{-1}$ ) cannot be owing to FcRn saturation. The $\mathrm{pH}$ in the extracelluar medium was set at $\mathrm{pH} 7.4$, suggesting the inhibition to takes place in intracellular compartments.

IgG1 and IgG3 compete for binding to FcRn and transport. Ober et al. demonstrated that, during sorting, the unbound FcRn molecules are routed away from the sorting endosomes ${ }^{15}$. This might explain the observed competition between IgG1 and IgG3 for FcRn binding and transport, because the number of FcRn molecules in intracellular compartments can also become limited under nonsaturating conditions. Indeed, we show that when the IgG3 concentration was kept constant at $10 \mu \mathrm{g} \mathrm{ml}^{-1}$, IgG1 concentrations as low as $1 \mathrm{ng} \mathrm{ml}^{-1}$ significantly reduced IgG3 transport (Fig. 2a). When IgG1 and IgG3 were mixed in equal amounts, the relative IgG3 transport was significantly reduced, compared with transport of IgG3 alone at high concentrations, but was unaffected at IgG concentrations of $10 \mathrm{ng} \mathrm{ml}^{-1} /$ subclass, when $\sim 4 \%$ of $1.25 \mu \mathrm{m}$ sorting endosomes ${ }^{15}$ would be expected to contain IgG (Fig. 2b). Remarkably, this concentration of IgG1 $\left(10 \mathrm{ng} \mathrm{ml}^{-1}\right)$ significantly inhibited a 1,000-fold excess of IgG3 (10 $\mu \mathrm{g} \mathrm{ml}^{-1}$, Fig. 2a). Similarly, soluble IgG1 was more efficient than IgG3 in inhibiting binding of soluble human FcRn (shFcRn) to IgG3-immobilized biosensor chips (Fig. 2c,d).

R435 in IgG interferes with binding to FcRn. When the differences in position 435 between IgG1 (Histidine) and IgG3 (Arginine) were modelled into the existing crystal structure ${ }^{21}$, it was clear that the longer side chain of arginine may potentially disrupt the tight fit of IgG in the FcRn-binding pocket (Fig. 3a,b). However, theoretically it may also convey a more favourable charge at $\mathrm{pH}$ 7.4, at which the net charge of histidine is neutral but arginine is positive ${ }^{22}$. IgG3 did indeed bind to shFcRn significantly better at pH 7.4, but IgG1 bound better at pH 6.0 as measured by ELISA (Supplementary Fig. S1) and by surface plasmon resonance (SPR) (Fig. 3c,d). We subsequently mutated IgG1 to include the IgG3-derived arginine residue and vice versa (IgG1-H435R and IgG3-R435H, respectively). These mutations resulted in the loss (IgG1-H435R) or gain (IgG3-R435H) of protein A binding (Supplementary Fig. S1).

Using shFcRn-coupled biosensor chips in SPR experiments, we noted that IgG1-H435R showed improved binding to FcRn at $\mathrm{pH}$ 7.4 compared with WT IgG1, but reduced binding affinity at acidic $\mathrm{pH}$. Likewise, binding of IgG3-R435H was reduced at $\mathrm{pH} 7.4$, but improved at acidic pH compared with WT IgG3 (Fig. 3c,d). The relative binding of both IgG subclasses and variants was however very weak at $\mathrm{pH} 7.4$, but increased steadily as the $\mathrm{pH}$ decreased, showing that shFcRn binding remained $\mathrm{pH}$-dependent for all variants.

The H435-containing IgG variants bound shFcRn with similar calculated affinities at pH 6.0 according to the bivalent ligand-binding model. However, IgG3 and IgG1-H435R had calculated kinetic dissociation constant $\left(\mathrm{K}_{D} \mathrm{~s}\right)$ that were, respectively, two times and $\sim 50 \%$ higher than WT IgG1 (Supplementary Fig. S2; Supplementary Table S1). Similar results were found using the heterogeneous ligand-binding model and by repeating the experiments with a reversed set-up, using IgG-coupled biosensor chips and the steadystate affinity model ${ }^{30}:$ shFcRn bound the strongest to IgG1-coupled chips at pH 6.0 whereas IgG1-H435R showed reduced binding. The affinity of shFcRn was improved for IgG3-R435H compared with IgG3 that showed the weakest affinity of all variants (Fig. 4).

The inhibition of IgG3 transport by IgG1 is due to R435. In agreement with the retained $\mathrm{pH}$-dependent FcRn-binding, the presence of histidine or arginine at position 435 did not influence the transport efficiency of either subclass when transported individually in 

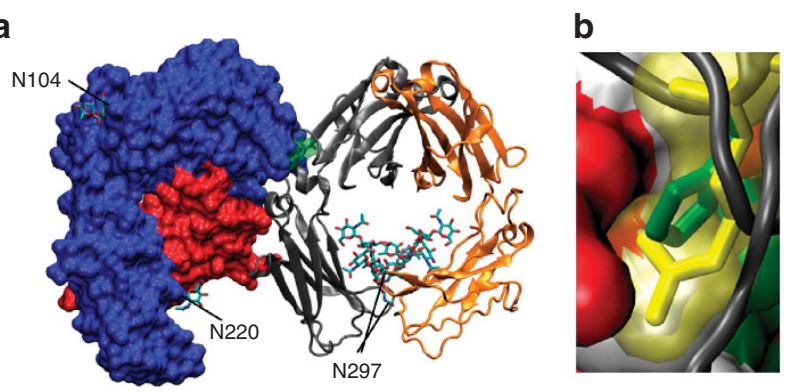

c
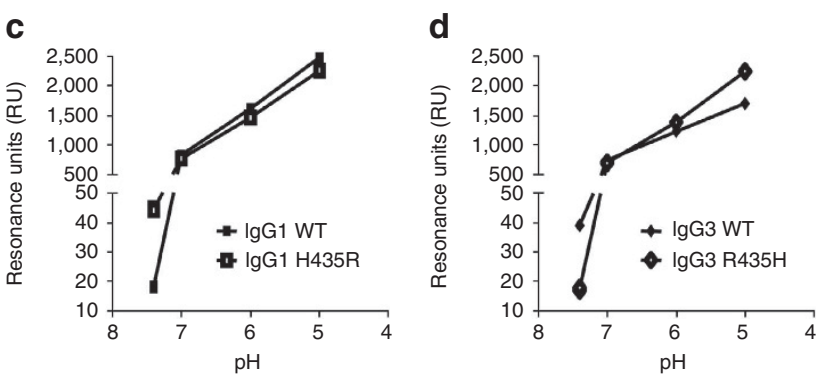

Figure 3 | The influence of the Histidine versus Arginine at position 435 on FcRn binding at different pH. (a) The crystal structure of FcRn with IgG-Fc part, showing the orientation of the amino acid 435 of IgG in yellow. $\mathrm{N}$-linked glycans are labelled according to their occurrence in FcRn and Fc. (b) A close-up showing the side chain of amino acid 435 of IgG (histidine, in green) in the binding pocket of $\mathrm{FcRn}$. When arginine (present at this position in lgG3) was modelled into this position (yellow), it protrudes into the FcRn surface area (non-polar residues in white, positively charged amino acids in blue, negatively charged residues in red and polar residues in green), suggesting steric hindrance. Histidine at this position alters its charge at pH 6.5 and lower (positive charge, resulting in FcRn binding) versus neutral $\mathrm{pH}$ (no charge, resulting in release of lgG from $\mathrm{FcRn})^{22}$. Arginine in this position, however, is positively charged at both low and neutral $\mathrm{pH}$, possibly resulting in better binding of $\operatorname{lgG} 3$ at neutral $\mathrm{pH}$. The crystallographic coordinates ${ }^{51}$ (accession 111A) were modelled using DeepView 4.03 (ref. 52) and VMD 1.9 (ref. 53). (c,d) The importance of this amino acid difference between $\lg \mathrm{g} 1$ and $\lg \mathrm{g} 3$ was tested biochemically by injecting $500 \mathrm{nM}$ recombinant IgG over shFcRn-coupled CM5 sensor chips at different pH. IgG3 (d) bound FcRn better than IgG1 (c) at neutral $\mathrm{pH}$, but the situation was reversed at acidic $\mathrm{pH}$. IgG1-H435R mutant gained IgG3-like characteristics, binding better at neutral $\mathrm{pH}$, but worse at low $\mathrm{pH}$ (c). Likewise, IgG3 behaved like IgG1 after replacing the R435 with $\mathrm{H} 435$, binding relatively worse at neutral $\mathrm{pH}$, but better at low $\mathrm{pH}(\mathbf{d})$, confirming that two opposing factors (steric hindrance versus charge) may contribute to the observed inhibition by IgG1 on FcRn-mediated IgG3 transport. The data in (c,d) are presented as individual data points connecting the means from two independent injections with a line.

A375-FcRn cells (Fig. 5a). When the amino acid at position 435 was mutated to an alanine, binding to protein A and shFcRn (Supplementary Fig. S1) as well as FcRn-dependent transport (Fig. 5a), was almost entirely abrogated both for IgG1 and IgG3. When mixed, IgG1-H435A did not inhibit transport of IgG3 (Fig. 5b), indicating that binding of IgG1 to FcRn was required for the observed inhibition of IgG3 transport. Importantly, IgG1-H435R (Fig. 5a) was also unable to inhibit IgG3 transport (Fig. 5b), indicating the arginine/ histidine difference between IgG3 and IgG1 to be important for IgG1mediated inhibition of IgG3 transport. In line with this, transport of IgG3-R435H was unaffected by the presence of any IgG1 variant (Fig. 5c). Remarkably, IgG3-R435H inhibited transport of IgG1H435R (Fig. 5c) to a similar extent as IgG1-WT inhibited transport of IgG3-WT (Fig. 5b). These results demonstrate that IgG-R435 can be transported by FcRn through cells, but that binding to, and transport by, FcRn is inhibited in the presence of H435-containing IgG.

Competition for FcRn leads to degradation of IgG3 in vitro. We next tested whether the reduced IgG3 transport in the presence of IgG1 was reflected in enhanced degradation during apical to basolateral transport. We therefore measured the recovery of different IgG variants from the apical and basolateral compartments (Fig. 6a). Up to $95 \%$ of $\operatorname{IgG} 1$ or IgG3 could be accounted for when transported alone. When mixed with IgG3, IgG1 recovery was unaffected, but only $60 \%$ of the initial amount of IgG3 was detected. However, when WT IgG1 and IgG3-R435H were mixed, recovery of both was 95\% (Fig. 6a), indicating that the decreased transport and enhanced loss of $\operatorname{IgG} 3$ in the presence of $\operatorname{IgG1}$ was solely due to the arginine at position 435.

IgG3 with $\mathrm{H} 435$ has an enhanced half-life in humans. The IgG3 $\mathrm{G} 3 \mathrm{~m}(s, t)$ allotype (Supplementary Fig. S3) contains a histidine at position 435, and can therefore be used to investigate the in vivo effect of the mutation. Although not commonly found in native Europeans, we found detectable levels in IVIg (Supplementary Fig. S4). ${ }^{31-34}$ We therefore investigated whether R435 can also cause the fast clearance of IgG3 in vivo, by analysing the serum persistence of this IgG3 allotype in sera of IVIg-treated X-linked agammaglobulinemic patients. Compared with the amounts found in the IVIg preparation itself, the amount of IgG1 and IgG2 relative to total IgG was unchanged in these patients four weeks after the last infusion of IVIg (Fig. 6b). As expected, the total serum IgG3 level, consisting mostly of R435 containing $\mathrm{G} 3 \mathrm{~m}(\mathrm{~b})$ and $\mathrm{G} 3 \mathrm{~m}(\mathrm{~g})$ allotypes in Europe ${ }^{31,32}$ (Supplementary Fig. S3), was significantly lowered, but significantly increased for the H435-containing IgG3 allotype $\mathrm{G} 3 \mathrm{~m}(s, t)$ (Fig. 6b). Thus, also in natural variants of $\operatorname{IgG} 3$, the presence of a histidine or an arginine at position 435 determines the catabolic rate in vivo.

Enhanced effector functions of $\mathrm{H} 435$ containing IgG3 in vivo. IgG3 binds $\mathrm{Clq}$ and activates Fc $\gamma \mathrm{R}$ more potently than any of the other IgG subclasses, but its therapeutic application has not yet been considered a viable option because of its short half-life ${ }^{4,35,36}$. We therefore compared the potential of the recombinant V-gene-matched IgG1, IgG3, IgG1-H435R and IgG3-R435H (directed against Streptococcus pneumoniae serotype 6) ${ }^{17,37}$ to stimulate Fc $\gamma \mathrm{R}$-mediated phagocytosis and mediate protection against pneumococcal pneumonia and bacteremia. IgG3 mediated more phagocytosis than IgG1, and neither isoallotypic alteration (IgG1 H435R/IgG3 R435H) significantly changed the capacity of the antibodies to mediate phagocytosis of pneumococci (Supplementary Fig. S5). We then passively immunized outbred NMRI mice $48 \mathrm{~h}$ before measuring the level of the human antibodies and intranasal challenge with virulent pneumococci. The serum persistence of all four $\operatorname{IgG}$ were measured before challenge that were found to be lower for IgG3 and IgG1-H435R than for IgG1 and IgG3-R435H (Fig. 7a). Bacteremia and pneumonia were determined $24 \mathrm{~h}$ later ${ }^{37}$. IgG1 and IgG3-R435H completely protected against bacteremia, whereas IgG3- and IgG1-H435Rtreated mice were partly protected (Fig. 7b). IgG3-R435H demonstrated a significantly better protection against pneumonia than IgG1 and IgG3, whereas IgG1-H435R was not protective (Fig. 7c). A complete clearance for a large fraction of the mice was only observed in the IgG3-R435H-treated group (Fig. 7b,c). The statistical analysis of the data in Figure $7 \mathrm{~b}$ is displayed in Table 1.

Collectively, these data reveal that the short half-life described for human IgG3 is due to the arginine in position 435 , that can be overcome by introducing an histidine present in this position in the $\mathrm{G} 3 \mathrm{~m}(s, t)$ allotypes and other $\operatorname{IgG}$ subclasses. The in vivo application of H435-IgG3 combines the properties of long half-life and strong effector functions that surpasses that of human IgG1. 
a
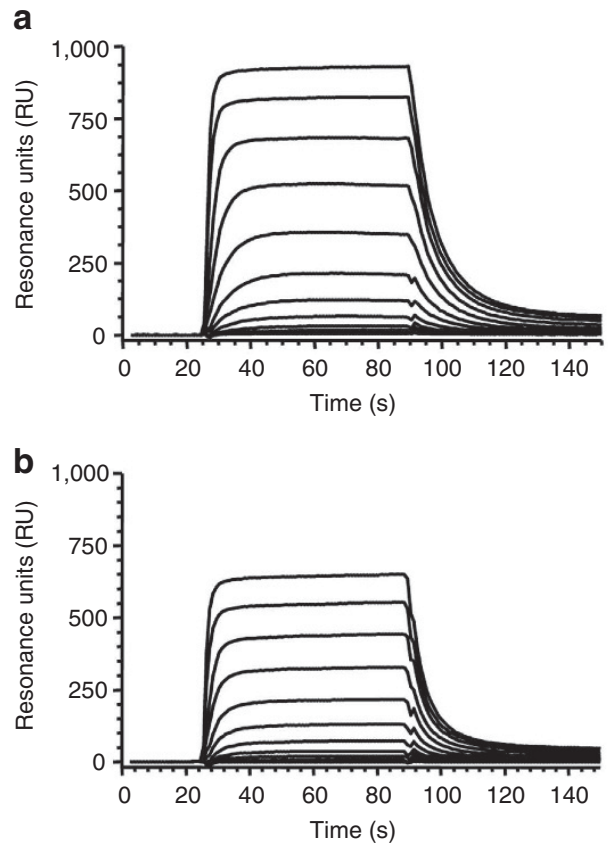

C
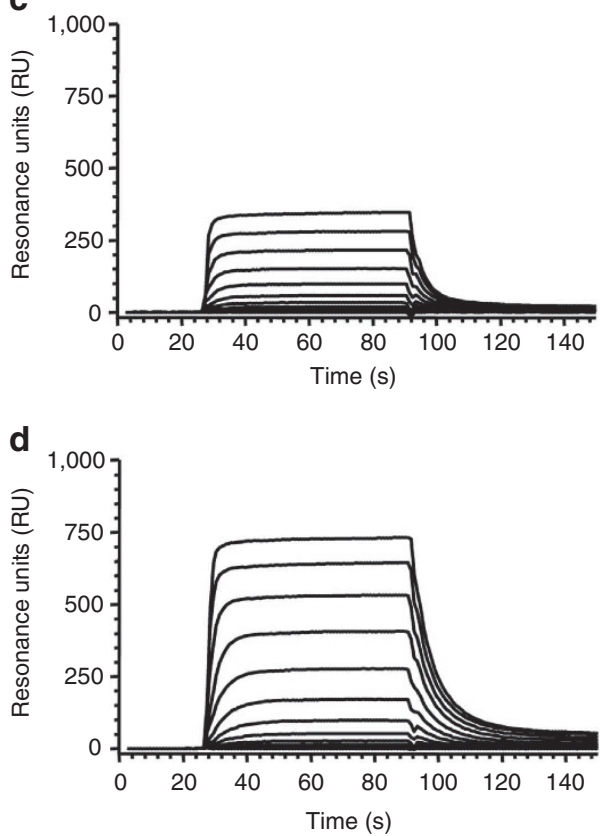

e

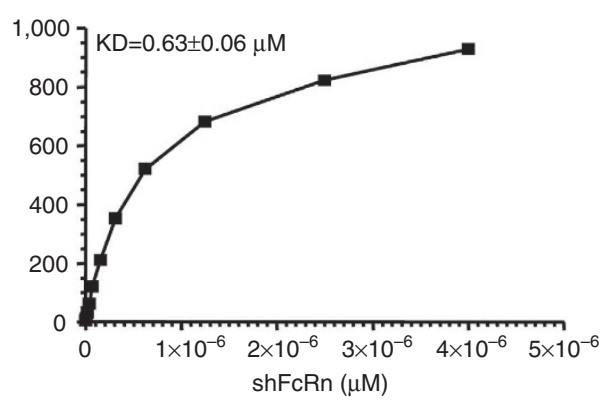

$\mathbf{f}$

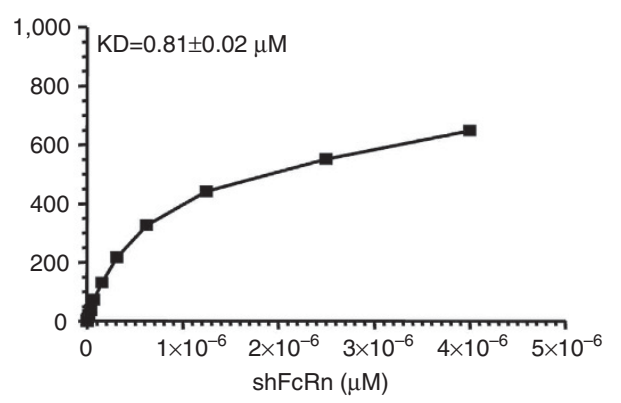

g

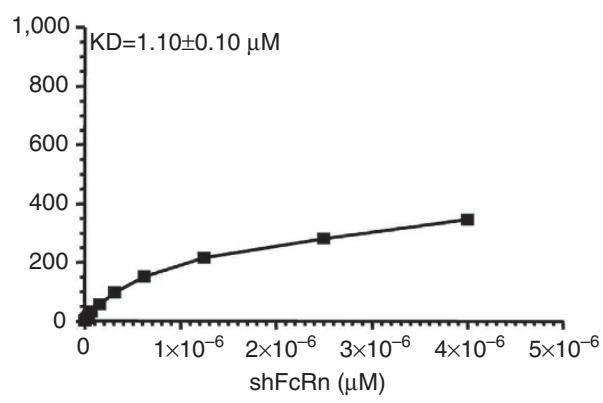

h

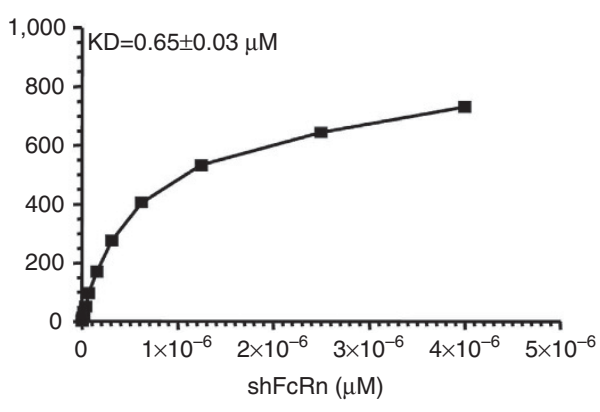

Figure 4 | Binding of the $\operatorname{lgG}$ at pH 6.0 is influenced by the amino acid at position 435. The different $\lg$ variants (IgG1, IgG1-H435R, $\lg G 3$, or IgG3-R435H) were immobilized to CM5 sensor chips and shFcRn injected at different concentrations (7-4,000 nM represented by different lines) at pH 6.0. The sensorgrams are shown in the left panels (a-d), and the corresponding equilibrium-binding responses versus shFcRn concentrations in the right panels (e-h), for IgG1 (a,e), IgG1-H435R (b,f), IgG3 (c, g), or IgG3-R435H (d,h). The calculated affinity constants are superimposed in the right panels as derived from steady-state binding model using the BIAevaluation software. FcRn shows a reduced affinity to IgG1 at pH 6.0 after mutating the $\mathrm{H}$ at position 435 to $\mathrm{R}$, and enhanced affinity to $\operatorname{lgG} 3$ after mutating the $\mathrm{R}$ at position 435 to $\mathrm{H}$. The data are representative from two independent injections.

\section{Discussion}

In this study, we investigated the binding of IgG3 to FcRn, which, although slightly lower and less $\mathrm{pH}$-dependent, was surprisingly similar to IgG1. In agreement with this, we found FcRn-mediated transport of purified IgG3 to be comparable to that of IgG1. These findings fail to explain the short half-life of IgG3 in humans. However, we found an unexpected inhibition of IgG3 transport in the presence of other IgG subclasses. This was because of intracellular competition for FcRn-mediated transport owing to a single amino acid difference in position 435 between IgG3(arginine) and the other IgG subclasses (histidine), which explains the shorter plasma half-life of IgG3. Mutating this arginine in IgG3 to histidine abrogated the inhibition by IgG1, without affecting the superior effector functions of IgG3.

FcRn-mediated transport and degradation were studied in hFcRntransfected human A375 cells and in the naturally FcRn-expressing human choriocarcinoma cellline JAR. The transport rates of purified IgG1 and IgG3 were similar for various IgG sources. However, in both cell types, the transport of IgG3 was inhibited by addition of IgG1. This inhibition was observed for myeloma IgG1 combined with IgG3 purified from Intramuscular Ig (IMIg), for V-gene-matched recom- 
a
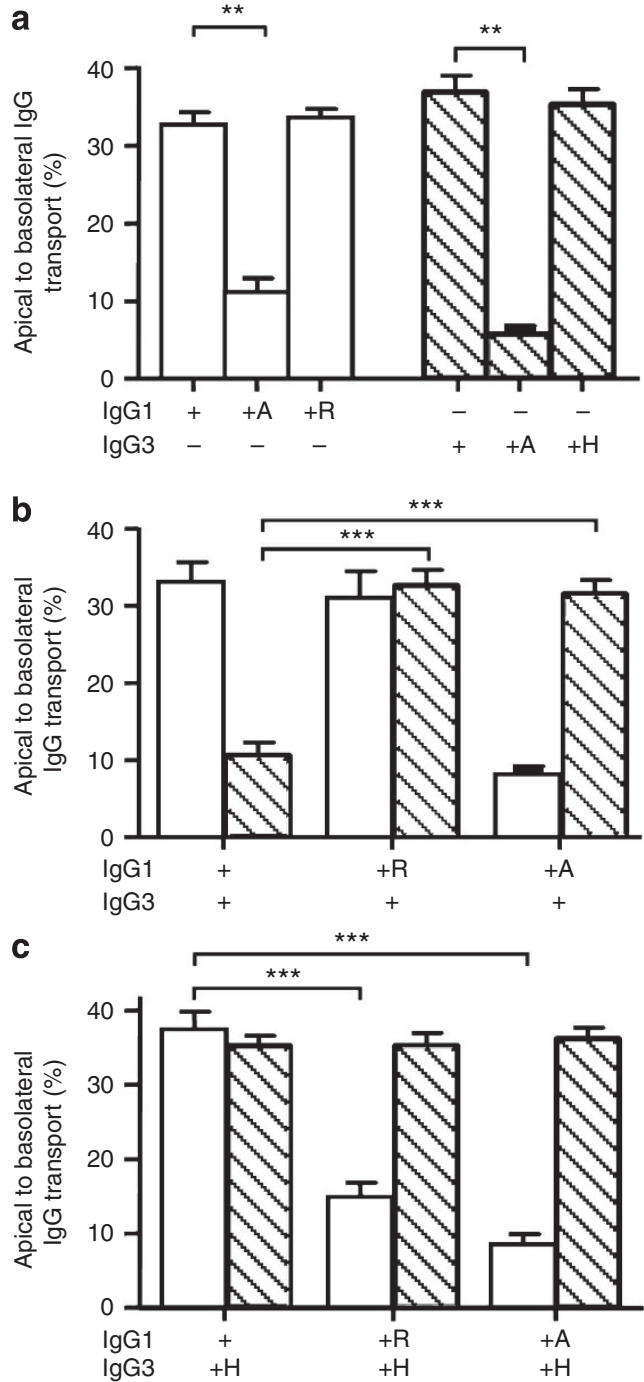

Figure 5 | Inhibition of IgG3 transport by IgG1 is due to R435 in lgG3.

(a) Mutating the amino acid at position 435 in $\operatorname{lgG} 1$ (H435) and in lgG3 (R435) to an alanine reduces transport, while exchanging the histidine native to $\lg G 1$ and the arginine native to $\lg G 3$ on each others backbone had no effect on their transport rate when offered separately to FcRntransfected A375 cells. (b) Whereas transport of IgG3-WT was inhibited in the presence of IgG1-WT, IgG1 bearing an alanine or an arginine at position 435 had no effect on IgG3 transport. (c) Transport of IgG3 with a histidine at position 435 was not inhibited by WT IgG1. When the amino acids found at position 435 in $\operatorname{lgG} 1$ and $\lg G 3$ were swapped, lgG1-H435R transport was inhibited by lgG3-R435H. (a-c) \pm indicate the presence or absence of $\operatorname{lgG}\left(10 \mu \mathrm{g} \mathrm{ml}^{-1}\right.$ per subclass), lgG1 is represented by open bars, IgG3 by hatched bars. The presence of mutated variants $(435 \mathrm{H}, 435 \mathrm{~A}$ and $435 \mathrm{R})$ is indicated by the corresponding letter. The data represent mean and standard deviation from three independent experiments. Transport of WT IgG was compared with transport of mutant IgG by one-way ANOVA with Dunnett's multiple comparison test and significance. ${ }^{\star \star} P \leq 0.01$; ${ }^{\star \star \star} P \leq 0.001$.

binant IgGs, and for IVIg in which the inhibition could be alleviated by a protein-A-based inhibitor of FcRn-IgG-binding (Z-domain) specific for IgG1, IgG2 and IgG4. The transport rate was independent of the IgG concentration indicating that an excess of $\mathrm{FcRn}$ over $\mathrm{IgG}$ will exist early after internalization. However, Ober et al. showed that there is a progressive depletion of FcRn after endo- or pinocytosis events as FcRn-containing vacuoles without IgG are routed away from sorting endosomes containing both IgG and FcRn ${ }^{15}$. Under these circum-
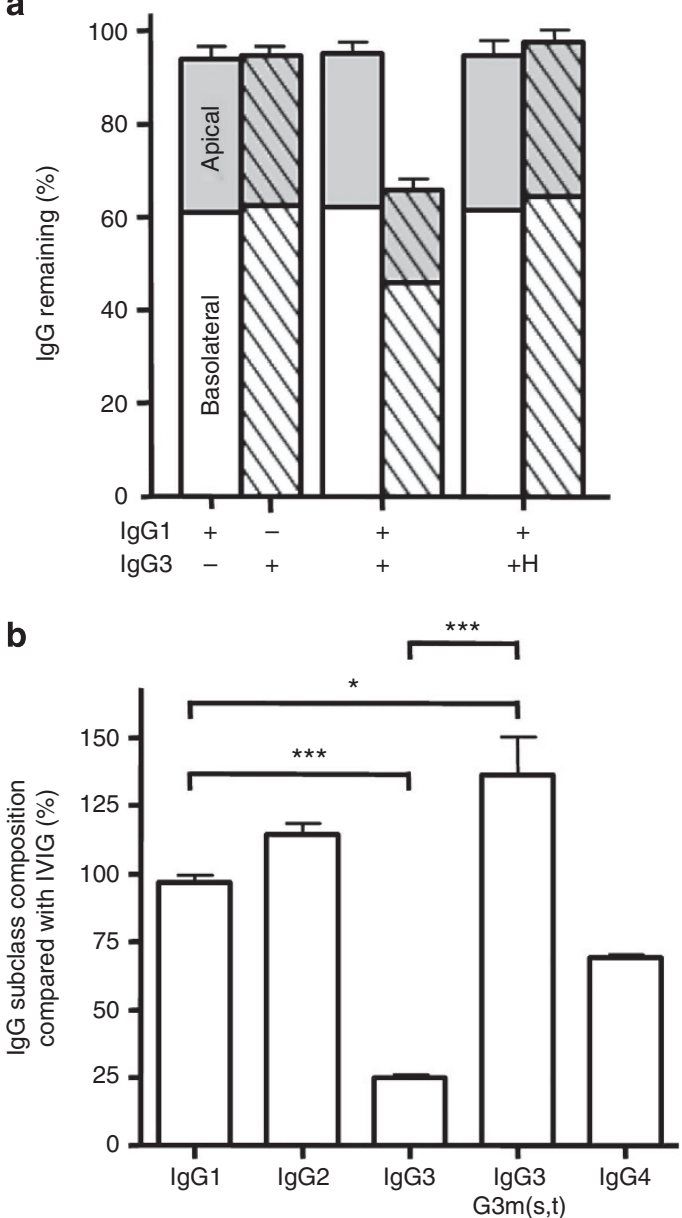

Figure 6 | H435-containing IgG3 has extended half-life in humans.

(a) Approximately $95 \%$ of the total lgG added to apical compartments of confluent A375-FcRn monolayers was recovered after $24 \mathrm{~h}$ from both the apical (grey) and the basolateral (white) compartments when the IgG1 (open bars) or lgG3 (hatched) were added individually $\left(10 \mu \mathrm{g} \mathrm{ml} \mathrm{l}^{-1}\right.$ per subclass). However, when the $\lg \mathrm{G} 1$ and $\lg \mathrm{G} 3$ were mixed in equal amounts, $\sim 65 \%$ of the initial lgG3 could be detected, suggesting lgG3 was degraded in the presence of $\lg G 1$. $\lg G 1$ recovery was similar to that found when no lgG3 was present. IgG3-R435H was not degraded in the presence of $\operatorname{lgG} 1$ as about $95 \%$ could be detected after $24 \mathrm{~h}$, similarly to lgG3 alone. The data represent mean and standard deviation from three independent experiments. (b) The relative concentration of IgG subclasses and the histidine- 435 containing IgG3 allotype $\mathrm{G} 3 \mathrm{~m}(\mathrm{~s}, \mathrm{t})$ in sera from agammaglobulinemic patients four weeks after their last treatment with IVIg compared with IgG subclass and $\mathrm{G} 3 \mathrm{~m}(\mathrm{~s}, t)$ levels found in the corresponding IVIg preparation. Data represent the average plus standard deviation calculated from at least three independent IgG subclass and allotype measurements performed on serum from three patients in (b). Statistical comparison was performed by one-way ANOVA followed by Tukey's multiple comparison test in (b). ${ }^{\star} P \leq 0.05$; ${ }^{\star \star}{ }^{\star} P \leq 0.001$. For simplicity, significant differences are only displayed for lgG1 compared with all subclasses, and between $\lg G 3$ total and $G 3 m(s, t)$ levels in (b).

stances, competition between IgG1 and IgG3 for formation of FcRnIgG complexes could favour IgG1 binding at low $\mathrm{pH}$ (refs $21,38,39$ ).

Significant transport inhibition of an excess of IgG3 was observed using very low concentrations of IgG1. Possibly this can be explained by recent findings showing that routing of IgG and FcRn does not take place inside discrete vesicles, but through elongated tubules and/or multiple recycling-endosomes tethered by tubules ${ }^{13,15,39}$. Here IgG probably binds FcRn in a stochiometry of 1:2, with two 
FcRn molecules on parallel membranes binding to one IgG molecule $^{21,39}$. If IgG in transit goes through multiple checkpoints in tubule-tethered recycling-endosomes ${ }^{15}$, then competition between IgG3 and IgG1 may occur sequentially. Differences in on-rates were the most prominent finding from the interaction kinetics studies, and this may at least partly explain the ability of low concentrations of IgG1 to inhibit IgG3-transport. Our findings that reduced IgG3 transport in the presence of IgG1 coincides with enhanced IgG3 degradation, support the hypothesis that this effect occurs after uptake and within developing lyso- and/or sorting endosomes.

We found that mutating position $435 \mathrm{in} \mathrm{IgG} \mathrm{to} \mathrm{an} \mathrm{alanine-reduced}$ transport almost to background levels and that IgG1-H435A was unable to inhibit FcRn-mediated IgG3 transport. Transportinhibition of IgG3 by IgG1 was completely reversed by swapping the amino acids normally present in IgG1 and IgG3 at position 435
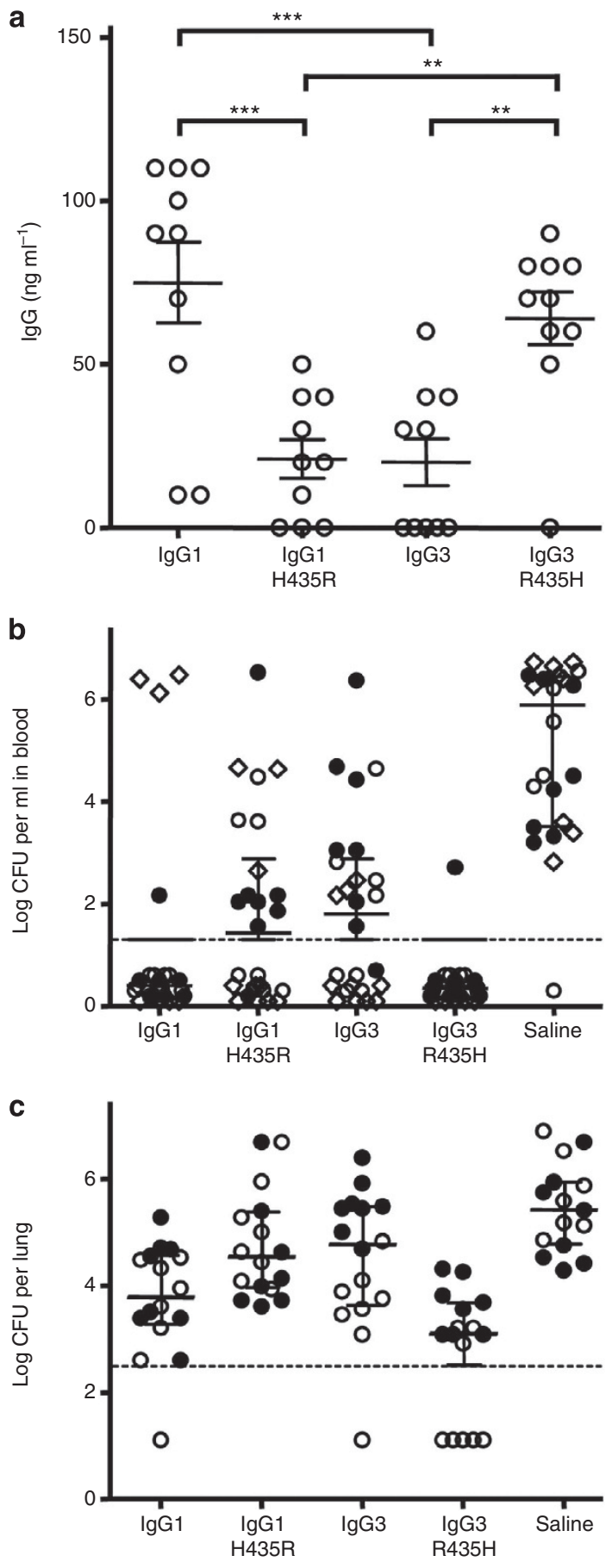

(resulting in IgG1-H435R and IgG3-R435H). This strongly indicated that human IgG1 is more adept at FcRn-mediated transport than IgG3, solely because of the R present at position 435 in IgG3. R435-containing IgG also bound $\operatorname{shFcRn}$ with a slightly lower affinity than H435-containing IgGs, and soluble IgG1 was better than IgG3 in inhibiting binding of shFcRn to solid-phase immobilized IgG3. This amino acid difference was found to affect IgG3 binding to FcRn in two ways-likely through reduced sensitivity to deprotonation resulting in enhanced binding at $\mathrm{pH} 7.4$, and through steric hindrance, causing decreased binding at $\mathrm{pH}$ 6.0, suggesting IgG3 to have a decreased competitiveness for $\mathrm{pH}$-dependent FcRn binding and release, and thus for the overall transport.

To study whether our in vitro observations also apply in vivo, we analysed sera from agammaglobulinemic patients who receive regular IVIg replacement therapy that contain both R435- and H435bearing IgG3 allotypes. We measured the relative IgG subclasses, and IgG3 allotype levels found in IVIg and sera, four weeks after the previous IVIg dose was given. In agreement with our in vitro findings, we found that these sera were enriched for the H435-containing IgG3 allotypes compared with total IgG3, demonstrating that the R435 is also responsible for the lack of competitive FcRn-mediated rescue and short half-life of IgG3 in humans. In accordance with this, we found the serum persistence of IgG1 and IgG3 R435H to be higher than that of IgG3 and IgG1 H435R in outbred NMRI mice. Furthermore, these findings show that the longer hinge of IgG3 does not lead to increased proteolytic degradation, which has been postulated to contribute to the lower half-life of IgG3 (ref. 4).

IgG3 binds FcyRIII and C1q with higher affinity than IgG1 and is capable of mediating considerably stronger effector functions, such as antibody-dependent cellular cytotoxicity, respiratory burst phagocytosis $1,36,40-42$, and complement-dependent cytotoxicity towards tumour cells ${ }^{3,43}$. This suggests IgG3 containing H435 to be a more effective candidate for in vivo immunotherapies. In an in vivo model of IgG-mediated protection against pneumococcal sepsis and bacteremia WT IgG3 gave comparable protection against pneumonia as IgG1. However, IgG3-R435H protected mice significantly better than either IgG1 or IgG3 against pneumococcal pneumonia, correlating both with its enhanced effector functions and half-life in mice, and confirming its therapeutic potential ${ }^{24}$.

Figure 7 | H435-containing lgG3 has increased serum persistence in mice and protects against pneumococcal pneumonia. (a) Serum IgG levels $48 \mathrm{~h}$ after injecting $1 \mu \mathrm{g}$ the IgG variants into outbred NMRI mice. lgG1-H435R and IgG3 show lower serum levels than lgG1 and IgG3R453H. ${ }^{\star \star} P \leq 0.01 ;{ }^{\star \star \star} P \leq 0.001$. (b) Three and (c) two experiments, showing outbred NMRI mice (8-10 per group per experiment) that were passively immunized intraperitoneally with $3 \mu \mathrm{g}$ (circles symbols) or $1 \mu \mathrm{g}$ (diamond) recombinant IgG anti-Streptococcus pneumoniae 6 or placebo $48 \mathrm{~h}$ before challenge with pneumococci of serotype $6 \mathrm{~A}$. Results from individual experiments are shown using the same symbol throughout (open or closed circles and diamonds). The number of bacteria found in (b) blood and (c) lungs $24 \mathrm{~h}$ after challenge are shown. (b) All but one mouse in the control group developed bacteremia, while all but four mice receiving WT IgG1 and all but one mouse receiving IgG3-R435H were completely protected. Approximately half of the mice receiving either $\lg \mathrm{G} 1-\mathrm{H} 435 \mathrm{R}$ or WT IgG3 developed bacteremia. (c) High numbers of bacteria were found in the lungs of control mice. All IgG-treated mice were significantly protected from lung infection, with lowered level of bacterial burden, with few mice in each group completely protected with no detectable bacteria. IgG3-R435H protected the mice significantly better than all other $\lg G$ variants, with five mice without detectable lung or blood infection. The dotted lines indicate the level of detection. Data in (a) represent the IgG levels in individual mice together with means and standard errors of means; data in $(\mathbf{b}, \mathbf{c})$ represent the bacterial load of individual mice together with medians (assuming the level of detection for individuals without detectable bacteria) and interquartile ranges, statistical comparison is tabulated in Table 1. 


\begin{tabular}{|c|c|c|c|c|}
\hline & IgG1-H435R & IgG3 & IgG3-R435H & Saline \\
\hline \multicolumn{5}{|l|}{ Blood } \\
\hline $\lg G 1$ & $0.0259^{\star}$ & 0.0144 & 0.1576 & $<0.0001$ \\
\hline lgG1-H435R & & 0.7267 & 0.0003 & $<0.0001$ \\
\hline $\operatorname{lgG3}$ & & & 0.0001 & $<0.0001$ \\
\hline IgG3-R435H & & & & $<0.0001$ \\
\hline \multicolumn{5}{|l|}{ Lung } \\
\hline $\lg G 1$ & 0.0215 & 0.0457 & 0.0139 & $<0.0001$ \\
\hline IgG1-H435R & & 0.8065 & $<0.0001$ & 0.0379 \\
\hline $\operatorname{lgG} 3$ & & & 0.0008 & 0.0379 \\
\hline IgG3-R435H & & & & $<0.0001$ \\
\hline
\end{tabular}

*Statistical comparison was performed with Mann-Whitney rank sum test.

In summary, IgG3 is known for both its superior effector functions compared with IgG1-the most commonly used isotype used for immunotherapy-but also for its short in vivo half-life ${ }^{4,5,35,36}$. We now show that FcRn can in fact rescue and transcytose IgG3 as efficiently as IgG1, but not in the presence of the other IgG subclasses. Our data support a model where IgG3 loses competition for FcRn binding and routing away from the lysosomal pathway, explaining its high catabolism in vivo. We demonstrate that this can be resolved by replacing the R435 with a histidine, as is the case in a rare natural variant. The epitope comprising the $\mathrm{H} 435$ in $\operatorname{G3m}(s, t)$ is an isoallotype that is found in all other IgG subclasses, and its detectable presence in IVIg has not been documented to cause adverse reactions in patients. The extended half-life of the IgG3-R435H variant has important implications for current and future antibody-based therapies aimed at achieving maximal effector functions.

\section{Methods}

Cell culture. Human choriocarcinoma cells (JAR, ATCC, VA) were grown in IMDM medium (Cambrex) and melanoma cells (A375, FcRn $\left.{ }^{-} \beta 2 \mathrm{~m}^{+}, \mathrm{ATCC}\right)$ in RPMI 1,640 medium (Invitrogen/Gibco), both supplemented with l-glutamin (300 $\mathrm{g} \mathrm{ml}^{-1}$, Invitrogen), penicillin (100 $\mathrm{U} \mathrm{ml}^{-1}$, PAA Laboratories), streptomycin $\left(100 \mu \mathrm{g} \mathrm{ml}^{-1}, \mathrm{PAA}\right)$ and $10 \%$ foetal calf serum (FCS).

A375-FcRn. Human FcRn messenger RNA was amplified after first strand cDNA synthesis by PCR using the forward 5'-GGATCCACCATGGGGGTCCCGCGGCC TCAGC- $3^{\prime}$ and reverse $5^{\prime}$-GAATTCTCAGGCGGTGGCTGGAATCAC- $3^{\prime}$ primers and ligated into pGEM-T (Promega, Madison, WI, USA). The BamHI-EcoRI fragment was subcloned into the pMX-puro vector (DNAX) and transfected together with a packaging vector into 293T-cells ${ }^{44}$. Supernatants were used to transduce A375 cells ${ }^{45}$. Expression of FcRn was confirmed by quantitative RT-PCR ${ }^{17}$.

IgG. IVIg was obtained from Sanquin. Pooled myeloma IgG1 was purified from three multiple myeloma patients by serum protein precipitation and depletion of other subclasses by sephadex-bound anti-subclass Ig. IgG3 was obtained from IMIg (Sanquin) after depleting other IgG with protein $\mathrm{A}$.

Recombinant IgG1, IgG3 and IgG1-H435A have been described before ${ }^{17,37}$ Recombinant IgG were produced in 293 Freestyle cells (Invitrogen) according to the manufacturer's instructions, and purified using HiTrap protein A (for H435containing IgGs) or protein $\mathrm{G}$ using the Acta Prime Plus system (GE Healthcare). IgG1-H435R, IgG3-R435A and IgG3-R435H were generated using the Quickchange Site-directed-mutagenesis kit (Stratagene) with the following and reverse complementary oligonucleotide primers:

\section{IgG1-H435R: GAGGCTCTGCACAACCGCTACACGCAGAAGAGCC}

IgG3-R435A:

\section{GAGGCTCTGCACAACGCCTACACGCAGAAGAGCC}

IgG3-R435H:

\section{GAGGCTCTGCACAACCACTACACGCAGAAGAGCC}

All mutations were confirmed by sequencing before expression. The endotoxin levels of the various batches was tested and confirmed to be very low or absent by measuring IL-6 production of monocyte-derived immature DC, as described in ref. 46.

$\mathbf{Z}$ domain. The gene encoding the $\mathbf{Z}$-domain was amplified by PCR from the vector pThio-His-ZZ ${ }^{47}$ using 5'-GGATCCGTAGACAACAAATTCAAC-3' (forward) and 5'-CTGCAGTTATTTCGGCGCCTGAGCATC-3' (reverse), and cloned into pGEM-T (Promega). The BamHI-NotI fragment was cloned into the pGEX 6.2 expression vector and expressed in Escherichia coli. The GST tag was removed using PreScision protease (GE healthcare) and the Z-domain eluted off the glutathione sepharose $4 \mathrm{~B}$ column (GE healthcare) by gravity flow and dialysed against PBS.

IgG transcytosis. $12 \mathrm{~mm}$ polycarbonate Transwell filters $(0.4 \mu \mathrm{m}$ pore size, Costar/ Corning) were inoculated with $5 \times 10^{5}$ cells, grown overnight to confluence, washed with PBS and medium replaced with fresh $1.5 \mathrm{ml}$ medium basolaterally and $0.5 \mathrm{ml}$ apically (IMDM at pH 7.4 with supplements as stated above). Mixtures of IgG contained $125 \mathrm{pg} \mathrm{ml}^{-1}$ streptavidin-HRP (Sanquin) to assess background transport. Apical to basolateral transport was calculated according to ([IgG $\left.]_{\text {basolateral }} \times 1.5 \mathrm{ml}\right) /$ $\left([\mathrm{IgG}]_{\text {input }} \times 0.5 \mathrm{ml}\right) \times 100 \%$. All experiments were performed in triplicate.

Surface plasmon resonance assays. All experiments were performed using a Biacore 3000 instrument, CM5 biosensor chips and amine coupling as described by the manufacturer (GE Healthcare). Injections were done using phosphate buffer ( $67 \mathrm{mM}$ phosphate buffer, $0.15 \mathrm{M} \mathrm{NaCl}, 0.05 \%$ Tween 20 ) at $\mathrm{pH} 6.0$. Kinetic evaluations were performed using immobilized shFcRn-GST $(\sim 1,000 \mathrm{RU})^{48,49}$, and IgG variants $(2-500 \mathrm{nM})$ injected. Alternatively, IgG was immobilized ( 1,500 RU) and shFcRn injected $(7-4,000 \mathrm{nM})$. For the competitive SPR assay, chips were coupled with $\operatorname{IgG}(\sim 1,300 \mathrm{RU})$ and $\operatorname{shFcRn}(100 \mathrm{nM})$ was injected alone or together with serial dilutions of IgG1 or IgG3. Binding at different $\mathrm{pH}$ was done in a phosphate buffer with $500 \mathrm{nM} \mathrm{IgG}$ injected over immobilized shFcRn ( 2.000 RU). All experiments took place at $20-60 \mu \mathrm{min}^{-1}, 25^{\circ} \mathrm{C}$, and HBS-P buffer (GE Healthcare) at $\mathrm{pH} 7.4$ was used for regeneration. Binding analyses were performed using the BIAevaluation wizard software 4.1.

Agammaglobulinemia patients. Serum samples from three agammaglobulinemia patients, taken 4 weeks after receiving IVIg, were assessed for IgG subclass levels which were expressed as relative IgG concentrations compared with that found in IVIg (Relative IgG levels at week $4(\%)=\left[\mathrm{IgG}_{S}\right]_{\mathrm{W}} /\left[\operatorname{IgG}_{T}\right]_{\mathrm{W}} /\left(\left[\mathrm{IgG}_{S}\right]_{\mathrm{IVIg}} /\right.$ $\left.\left[\operatorname{IgG}_{T}\right]_{\mathrm{IVIg}}\right) \times 100 \%$, where W4 stands for serum at week 4 after IVIg injection, $S$ for Subclass and $T$ for Total). Serum samples were anonymous rest material acquired during routine IgG-evaluation of these patients, and, therefore, did not require a Medical Ethical Committee evaluation according to the institutional Committee of the Academic Medical Centre of the University of Amsterdam.

IgG quantification. IgG subclass concentrations in sera were determined by Nephelometry (Behringer Nephelometer II, Behringer diagnostics). For other experiments, IgG concentrations were determined by sandwich ELISA using subclass specific mouse monoclonal antibodies (IgG1:MH161-1; IgG3:MH163-1, Sanquin) or allotype-specific monoclonal anti G3m $(s, t)^{32}$ (1.5A10, Sanquin, Supplementary Fig. S4) for capture. Mouse-anti-IgG-HRP (Southern Biotech) was used for detection in all assays except the G3m $(s, t)$ ELISA where mouse anti-IgG3-HRP (MH163-1, Sanquin) was used. Concentration was read of standard curves made using the same IgG preparations used for transport or to treat patients.

Passive protection. The pneumococcal infection model has been described before $^{37,50}$. In brief, outbred NMRI mice (Taconic) were passively immunized intraperitoneally with $3 \mu \mathrm{g}$ of recombinant IgG (diluted in $200 \mu \mathrm{l}$ PBS) or saline only, $48 \mathrm{~h}$ before challenge with a virulent pneumococcal strain of serotype $6 \mathrm{~A}$. Mice were anesthetized and challenged intranasally with $2 \times 10^{7} \mathrm{CFU}$ pneumococci in $50 \mu \mathrm{l}$ sterile $0.9 \%$ saline. Twenty-four hours after challenge, the mice were killed and bacterial load evaluated by colony counting as described in refs 37,50 . All in vivo studies complied with the Animal Experimental Committee of Iceland According to the Act on Animal Welfare no 15/1994.

Statistical analysis and data sets. All data represent the mean and standard deviation of at least three independent experiments. All transcytosis assays consisted of three replicates. GraphPad Prism version 4.00 for Windows (GraphPad Software) was used for all statistical analysis. Significance was set at $P \leq 0.05$, and the level of significance is indicated on all figures as ${ }^{\star} P \leq 0.05 ;{ }^{* *} P \leq 0.01 ;{ }^{* * *} P \leq 0.001$.

\section{References}

1. Bruhns, P. et al. Specificity and affinity of human Fcgamma receptors and their polymorphic variants for human IgG subclasses. Blood 113, 3716-3725 (2009).

2. Salfeld, J. G. Isotype selection in antibody engineering. Nat. Biotechnol. 25, 1369-1372 (2007).

3. Natsume, A. et al. Engineered antibodies of $\operatorname{IgG} 1 / \operatorname{IgG} 3$ mixed isotype with enhanced cytotoxic activities. Cancer Res. 68, 3863-3872 (2008).

4. Jefferis, R. Antibody therapeutics: isotype and glycoform selection. Expert Opin. Biol. Ther. 7, 1401-1413 (2007).

5. Morell, A., Terry, W. D. \& Waldmann, T. A. Metabolic properties of IgG subclasses in man. J. Clin. Invest. 49, 673-680 (1970).

6. Story, C. M., Mikulska, J. E. \& Simister, N. E. A major histocompatibility complex class I-like Fc receptor cloned from human placenta: possible role in transfer of immunoglobulin G from mother to fetus. J. Exp. Med. 180, 2377-2381 (1994). 
7. Simister, N. E. \& Mostov, K. E. An Fc receptor structurally related to MHC class I antigens. Nature 337, 184-187 (1989).

8. Raghavan, M., Bonagura, V. R., Morrison, S. L. \& Bjorkman, P. J. Analysis of the $\mathrm{pH}$ dependence of the neonatal $\mathrm{Fc}$ receptor/immunoglobulin $\mathrm{G}$ interaction using antibody and receptor variants. Biochemistry 34, 14649-14657 (1995).

9. Vaughn, D. E. \& Bjorkman, P. J. Structural basis of $\mathrm{pH}$-dependent antibody binding by the neonatal Fc receptor. Structure 6, 63-73 (1998)

10. Wu, Z. \& Simister, N. E. Tryptophan- and dileucine-based endocytosis signals in the neonatal Fc receptor. J. Biol. Chem. 276, 5240-5247 (2001).

11. McCarthy, K. M. et al. Effects of mutations in potential phosphorylation sites on transcytosis of FcRn. J. Cell Sci. 114, 1591-1598 (2001).

12. Newton, E. E., Wu, Z. \& Simister, N. E. Characterization of basolateral targeting signals in the neonatal Fc receptor. J. Cell Sci. 118, 2461-2469 (2005).

13. He, W. et al. FcRn-mediated antibody transport across epithelial cells revealed by electron tomography. Nature 455, 542-546 (2008).

14. Lencer, W. I. \& Blumberg, R. S. A passionate kiss, then run: exocytosis and recycling of IgG by FcRn. Trends Cell Biol. 15, 5-9 (2005).

15. Ober, R. J., Martinez, C., Vaccaro, C., Zhou, J. \& Ward, E. S. Visualizing the site and dynamics of IgG salvage by the MHC class I-related receptor, FcRn. J. Immunol. 172, 2021-2029 (2004).

16. Mi, W. et al. Targeting the neonatal fc receptor for antigen delivery using engineered fc fragments. J. Immunol. 181, 7550-7561 (2008).

17. Vidarsson, G. et al. FcRn: an IgG receptor on phagocytes with a novel role in phagocytosis. Blood 108, 3573-3579 (2006).

18. Qiao, S. W. et al. Dependence of antibody-mediated presentation of antigen on FcRn. Proc. Natl Acad. Sci. USA 105, 9337-9342 (2008).

19. Baker, K. et al. Neonatal Fc receptor for IgG (FcRn) regulates crosspresentation of IgG immune complexes by CD8-CD11b+ dendritic cells.. Proc Natl Acad. Sci. USA 108, 9927-9932 (2011).

20. Chaudhury, C. et al. The major histocompatibility complex-related Fc receptor for IgG $(\mathrm{FcRn})$ binds albumin and prolongs its lifespan. J. Exp. Med. 197, 315-322 (2003).

21. Burmeister, W. P., Huber, A. H. \& Bjorkman, P. J. Crystal structure of the complex of rat neonatal Fc receptor with Fc. Nature 372, 379-383 (1994).

22. Shimizu, A. et al. H NMR studies of the Fc region of human IgG1 and IgG3 immunoglobulins: assignment of histidine resonances in the $\mathrm{CH} 3$ domain and identification of IgG3 protein carrying G3m(st) allotypes. Mol. Immunol. 20, 141-148 (1983).

23. Dall'Acqua, W. F. et al. Increasing the affinity of a human IgG1 for the neonatal Fc receptor: biological consequences. J. Immunol. 169, 5171-5180 (2002).

24. Kim, J. K. et al. Mapping the site on human IgG for binding of the MHC class I-related receptor, FcRn. Eur. J. Immunol. 29, 2819-2825 (1999).

25. Kenanova, V. et al. Tailoring the pharmacokinetics and positron emission tomography imaging properties of anti-carcinoembryonic antigen single-chain Fv-Fc antibody fragments. Cancer Res. 65, 622-631 (2005).

26. West, A. P. Jr. \& Bjorkman, P. J. Crystal structure and immunoglobulin G binding properties of the human major histocompatibility complex-related Fc receptor. Biochemistry 39, 9698-9708 (2000).

27. Hassan, M. S., Abedi-Valugerdi, M., Lefranc, G., Hammarstrom, L. \& Smith, C. I. Biological half-life of normal and truncated human IgG3 in scid mice. Eur. J. Immunol. 21, 1319-1322 (1991).

28. Braisted, A. C. \& Wells, J. A. Minimizing a binding domain from protein A. Proc. Natl Acad. Sci. USA 93, 5688-5692 (1996).

29. Raghavan, M., Chen, M. Y., Gastinel, L. N. \& Bjorkman, P. J. Investigation of the interaction between the class I MHC-related Fc receptor and its immunoglobulin G ligand. Immunity 1, 303-315 (1994).

30. Andersen, J. T., Daba, M. B., Berntzen, G., Michaelsen, T. E. \& Sandlie, I. Cross-species binding analyses of mouse and human neonatal Fc receptor show dramatic differences in immunoglobulin $\mathrm{G}$ and albumin binding. J. Biol. Chem. 285, 4826-4836 (2010)

31. Steinberg, A. G. Globulin polymorphisms in man. Annu. Rev. Genet. 3, 25-52 (1969).

32. Martensson, L., van Loghem, E., Matsumoto, H. \& Nielsen, J. Gm(s) and $\mathrm{Gm}(\mathrm{t})$ : genetic determinants of human gamma-globulin. Vox Sang. 11, 393-418 (1966).

33. Lefranc, M. P. et al. Gene conversion in human immunoglobulin gamma locus shown by unusual location of IgG allotypes. FEBS Lett. 196, 96-102 (1986).

34. Pan, Q. \& Hammarstrom, L. Molecular basis of IgG subclass deficiency. Immunol. Rev. 178, 99-110 (2000).

35. Redpath, S., Michaelsen, T. E., Sandlie, I. \& Clark, M. R. The influence of the hinge region length in binding of human IgG to human Fcgamma receptors. Hum. Immunol. 59, 720-727 (1998).

36. Vidarsson, G. et al. Activity of human IgG and IgA subclasses in immune defense against Neisseria meningitidis serogroup B. J. Immunol. 166, 6250-6256 (2001).

37. Saeland, E. et al. Central role of complement in passive protection by human IgG1 and IgG2 anti-pneumococcal antibodies in mice. J. Immunol. 170, 6158-6164 (2003).

38. Tesar, D. B., Tiangco, N. E. \& Bjorkman, P. J. Ligand valency affects transcytosis, recycling and intracellular trafficking mediated by the neonatal $\mathrm{FC}$ receptor. Traffic 7, 1127-1142 (2006).
39. He, Y., Jensen, G. J. \& Bjorkman, P. J. Nanogold as a specific marker for electron cryotomography. Microsc. Microanal. 15, 183-188 (2009).

40. Aase, A. \& Michaelsen, T. E. Opsonophagocytic activity induced by chimeric antibodies of the four human IgG subclasses with or without help from complement. Scand. J. Immunol. 39, 581-587 (1994).

41. Brouwers, H. A. et al. Maternal antibodies against fetal blood group antigens A or B: lytic activity of IgG subclasses in monocyte-driven cytotoxicity and correlation with $\mathrm{ABO}$ haemolytic disease of the newborn. Br. J. Haematol. 70, 465-469 (1988).

42. Huizinga, T. W. et al. Binding characteristics of dimeric IgG subclass complexes to human neutrophils. J. Immunol. 142, 2359-2364 (1989).

43. Bruggemann, M. et al. Comparison of the effector functions of human immunoglobulins using a matched set of chimeric antibodies. J. Exp. Med. 166, 1351-1361 (1987).

44. Onishi, M. et al. Applications of retrovirus-mediated expression cloning. Exp. Hematol. 24, 324-329 (1996).

45. Cassard, L. et al. Expression of low-affinity Fc gamma receptor by a human metastatic melanoma line. Immunol. Lett. 75, 1-8 (2000).

46. Kikkert, R., de Groot, E. R. \& Aarden, L. A. Cytokine induction by pyrogens: comparison of whole blood, mononuclear cells, and TLR-transfectants. J. Immunol. Methods 336, 45-55 (2008).

47. Zhao, Y. et al. Multi-antigen immunization using IgG binding domain $\mathrm{ZZ}$ as carrier. Vaccine 23, 5082-5090 (2005)

48. Andersen, J. T. et al. A strategy for bacterial production of a soluble functional human neonatal Fc receptor. J. Immunol. Methods 331, 39-49 (2008).

49. Andersen, J. T. et al. Ligand binding and antigenic properties of a human neonatal Fc receptor with mutation of two unpaired cysteine residues. FEBS J. 275, 4097-4110 (2008).

50. Saeland, E., Vidarsson, G. \& Jonsdottir, I. Pneumococcal pneumonia and bacteremia model in mice for the analysis of protective antibodies. Microb. Pathog. 29, 81-91 (2000)

51. Martin, W. L., West, A. P. Jr., Gan, L. \& Bjorkman, P. J. Crystal structure at 2.8 A of an FcRn/heterodimeric Fc complex: mechanism of $\mathrm{pH}$-dependent binding. Mol. Cell 7, 867-877 (2001).

52. Guex, N. \& Peitsch, M. C. SWISS-MODEL and the Swiss-PdbViewer: an environment for comparative protein modeling. Electrophoresis 18, 2714-2723 (1997).

53. Humphrey, W., Dalke, A. \& Schulten, K. VMD: visual molecular dynamics. J. Mol. Graph. 14, 33-38 (1996).

\section{Acknowledgements}

We thank Dr Rob Aalberse, Dr Lucien Aarden, Dr Dirk Roos, Dr Réne van Lier, Dr Gerda de Lange for helpful discussion; Dr Taco Kuipers for help in obtaining samples from agammaglobulinemic patients; Dr Peter van Ede for the anti-G3m $(s, t)$ monoclal antibody, Dr Wim van Esch for myeloma IgG1 and IgG3 isolated from IMIg, and Ed Nieuwenhuis and colleagues for measuring IgG levels by Nephelometry. Ir. N.M.S. was supported by an NWO Aspasia grant number 015.001.083. G.V. was supported by an LSBR grant, number 0426. J.T.A. was supported by the Norwegian Research Council, grant number 179573/V40, and by the South-Eastern Norway Regional Health Authority, grant number 39375

\section{Author contributions}

N.M.S. performed all transwell experiments and ELISA on patient sera. I.S., G.V. and J.T.A. designed biosensor experiments. J.T.A. performed all biosensor and ELISAs with recombinant FcRn. G.V., I.J., and S.P.B. designed mouse experiements and S.P.B. performed them. A.M.S., R.V. and G.V. generated recombinant and mutant IgG1 and IgG3 and the A375-FcRn transfectant. J.G. performed phagocytosis experiments. G.V., Y.Z. and N.M.S. generated the Z-domains. M.K. and M.d.H. set up transwell experiments. M.d.H., C.E.v.d.S. and G.V. conceived the project. C.E.v.d.S., N.M.S. and G.V. developed the hypothesis and designed the experiments. C.E.v.d.S and G.V. coordinated and directed the project. The manuscript was written by N.M.S. and G.V. The latter discussed the hypothesis with N.M.S., A.M.S., S.P.B. M.d.H., I.J., J.T.A. I.S. who also helped with data interpretation, and writing the manuscript.

\section{Additional information}

Supplementary Information accompanies this paper at http://www.nature.com/ naturecommunications

Competing financial interests: The authors declare no competing financial interests.

Reprints and permission information is available online at http://npg.nature.com/ reprintsandpermissions

How to cite this article: Stapleton, N. M. et al. Competition for FcRn-mediated transport gives rise to short half-life of human IgG3 and offers therapeutic potential. Nat. Commun. 2:599 doi: 10.1038/ncomms1608 (2011)

License: This work is licensed under a Creative Commons Attribution-NonCommercialNoDerivative Works 3.0 Unported License. To view a copy of this license, visit http:// creativecommons.org/licenses/by-nc-nd/3.0/ 